\title{
Characterization of Fatty Acids in the Sebum of Goats According to Sex and Age
}

\author{
Takeyoshi Sugiyama, Hitoshi Matsuura, Hiroshi Sasada, ${ }^{*}$ \\ Junji MASAKI* and Kyohei YamaSHITA \\ Department of Agricultural Chemistry and *Department of Animal Science, \\ Faculty of Agriculture, Tohoku University, \\ Sendai 980, Japan
}

Received June 6, 1986

\begin{abstract}
Fatty acids in the sebum of goats were analyzed. 4-Ethyl fatty acids were the major components in mature males, while iso-, anteiso-, and normal fatty acids were the major ones in females. Normal fatty acids predominated in the sebum of immature goats of both sexes. There was no significant difference in the constituents between Saanen and Japanese native breeds.
\end{abstract}

A goat is a seasonal breeder and the mature male releases a specific odor from its hair, mainly at the head and neck in the breading season. This odor is male-specific and may be an attractant to the opposite sex. ${ }^{1}$ In our previous paper, ${ }^{2)}$ we reported the extraction, separation, and structural elucidation of the main components of buck-released odors in Japanese native breeds; they were novel 4ethyl fatty acids, (4-ethyl-octanoic, -decanoic, -dodecanoic, -tetradecanoic, and -hexadecanoic acids). 4-Ethyl-octanoic acid has the most intense odor responsible for buck-specific odors.

Although these unusual fatty acids seem to occur only in the adult male, comparative analysis on the fatty acids in the sebum of both sexes is necessary for investigation of the roles and effects of these substances on the sexual behavior of goats.

In this paper, we report the analytical study of fatty acid components in the sebum of immature and mature goats of both sexes.

\section{MATERIALS AND METHODS}

Goat. Male and female goats of the following ages were analyzed: Saanen breed: two juveniles (1.5 2 months of age), two young adults $(9.3 \sim 9.7$ months, the same individuals as above) and four adults ( $2 \sim 4$ years $)$
Japanese native breed: four adults ( $2 \sim 4$ years). Samplings for analyses were done in the breeding season except for juveniles.

Methyl esters of standard fatty acids. The following fatty acids were esterified with ethereal diazomethane:

4-ethyl- and 4-methyl-fatty acids: synthesized as previous described. ${ }^{2}$ Normal fatty acids: purified from commercial preparations.

A mixture of odd-carbon-numbered anteiso- and evencarbon-numbered iso-acids: though each of these acids had been isolated from lanolin, ${ }^{3)}$ a mixture of methyl esters was prepared from a similar source by the following method. A crude hydrolyzate of lanolin ${ }^{3)}$ was methylesterified with diazomethane to give a mixture of methyl esters which was chromatographed on silica gel. Elution with hexane-ethyl acetate $(20: 1)$ gave a mixture of less polar methyl esters which was further purified by vacuum distillation. A fraction distilled below $150^{\circ} \mathrm{C}(2 \mathrm{mmHg})$ was collected, analyzed by GC-MS, and found to be a mixture of methyl esters of normal fatty acids $\left(\mathrm{C}_{10}-\mathrm{C}_{20}\right)$, even-carbon-numbered iso-acids $\left(\mathrm{C}_{10}-\mathrm{C}_{20}\right)$, and oddcarbon-numbered anteiso-acids $\left(\mathrm{C}_{11}-\mathrm{C}_{19}\right)$.

Extraction and fractionation of fatty acids in the sebum. The hair of the head and neck of individual goats was cut off and extracted with ether. The extract was concentrated and partitioned between ether and $5 \%$ sodium carbonate solution when the free acids were detected in the extract by thin layer chromatography. The above aqueous layer was acidified and re-extracted with ether, and the extract was subjected to analysis of free acids. The neutral fraction was concentrated to dryness and hydrolyzed with alcoholic potassium hydroxide or $5 \%$ hydrochloric acid-dioxane 
(1:1). The recovered acidic fractions were esterified with diazomethane and analyzed by gas chromatography.

Conditions of gas chromatography.

Model: Hitachi Gas Chromatograph type 163 equipped with flame ionization detectors. Column: $2 \mathrm{~m} \times 3 \mathrm{~mm}$ glass column packed with $4 \%$ silicone SE-30 on Chromosorb W and a similar column packed with $5 \%$ DEGS on Diasolid M. Nitrogen flow rate: $20 \mathrm{ml} / \mathrm{min}$. Column temperature: linear programming from 140 to $200^{\circ} \mathrm{C}$ or $190^{\circ} \mathrm{C}$ isothermal.

\section{RESULTS AND DISCUSSION}

Yields of ether extracts per hair weight were $1 \sim 6 \%$ (adult males), $0.5 \sim 2 \%$ (adult females) and approximately $1 \%$ (juveniles). In most of these extracts, free sterols and their esters were detected on thin layer chromatograms developed with hexane-ethyl acetate $(4: 1)$. They were monitored by the peculiar coloration on heating the TLC plates after spraying with dilute sulfuric acid (cholesterol was used as a reference). Free acids were present in consider-

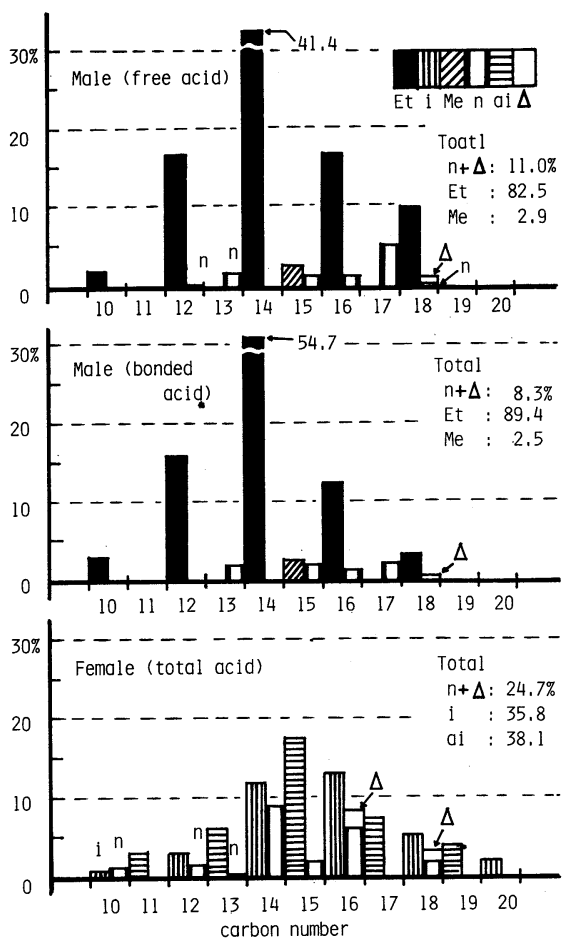

Fig. 1. Fatty Acids in the Sebum of Mature Goats (Japanese native breed).

Abbreviations: $\mathrm{n}$, normal fatty acids; $\triangle$, unsaturated fatty acids; ai, anteiso-acids; i, iso-acids; Et, 4-ethyl fatty acids; Me, 4-methyl fatty acids. able amounts in the extract of adult males, and in very small amounts in those of females and juveniles. Therefore, the latter extracts were directly saponified. The proportions of total acidic fractions in the extracts were 20 to $50 \%$.

Typical patterns of fatty acids in the sebum of males and females are illustrated in Fig. 1 (Japanese native breed), Fig. 2, and Fig. 3 (Saanen breed). The proportions of each fatty acid are displayed by the classification with respect to the type of compound. There was no significant difference in qualities between the free-acid and bonded-acid fractions of both breeds. The main acidic components in the sebum of mature males were 4-ethyl fatty acids in both breads, in agreement with the previous observations. $^{2)}$

The acidic constituents of mature females, however, were clearly different from those of mature males, irrespective of breeds as shown in Fig. 1 and Fig. 3. The percentage of normal fatty acids was higher and most of the branched fatty acids, which were at least $45 \%$ of
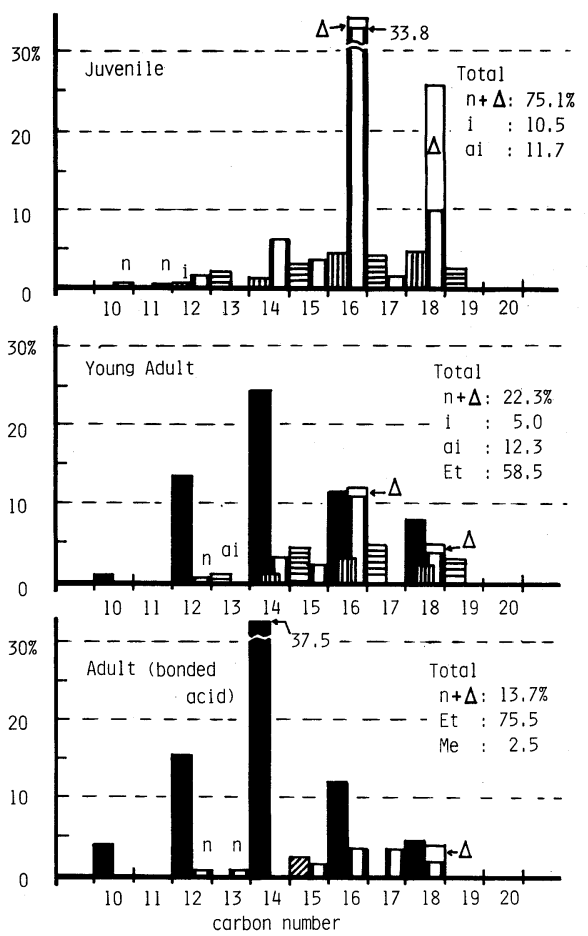

FIG. 2. Fatty Acids in the Sebum of Male Saanen Breed. Abbreviations, see Fig. 1. 


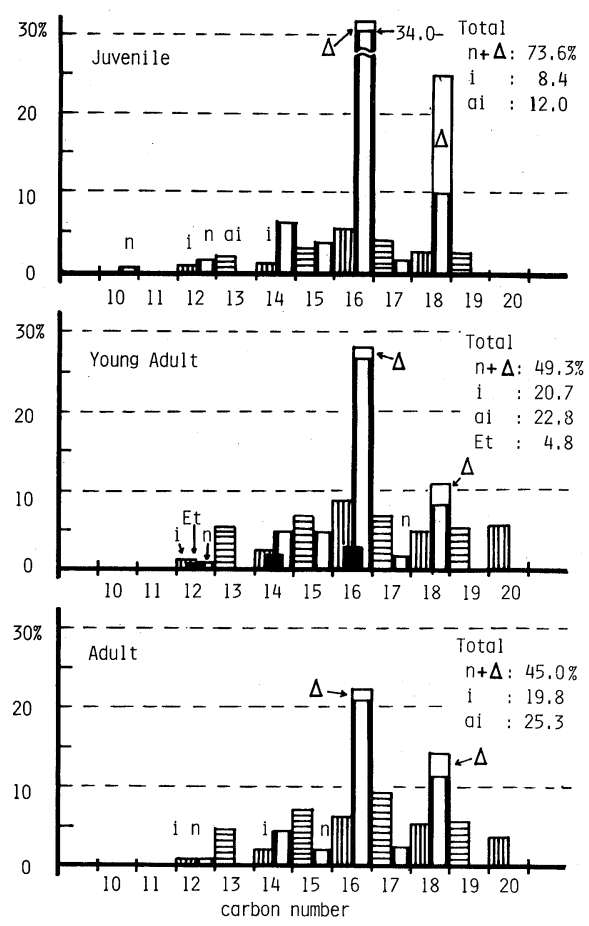

FIG. 3. Fatty Acids in the Sebum of Female Saanen Breed.

Abbreviations, see Fig. 1.

$$
\begin{aligned}
& \text { Normal fatty acid } \\
& \mathrm{CH}_{3}\left(\mathrm{CH}_{2}\right)_{n} \mathrm{COOH}
\end{aligned}
$$

Anteiso-acid
$\mathrm{CH}_{3}$
$\mathrm{CH}_{3} \mathrm{CH}_{2}-\mathrm{CH}\left(\mathrm{CH}_{2}\right)_{2 n} \mathrm{COOH}$

4-Ethyl fatty acid

$\mathrm{CH}_{3}\left(\mathrm{CH}_{2}\right)_{2 n+1} \stackrel{\text { C }}{\mathrm{CH}}\left(\mathrm{CH}_{2}\right)_{2} \mathrm{COOH}$
Unsaturated fatty acid $\mathrm{CH}_{3}\left(\mathrm{CH}_{2}\right)_{m} \mathrm{CH}=\mathrm{CH}\left(\mathrm{CH}_{2}\right)_{n} \mathrm{COOH}$

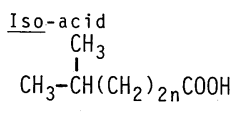

4-Methyl fatty acid $\mathrm{CH}_{3}$

$\mathrm{CH}_{3}\left(\mathrm{CH}_{2}\right)_{2 \mathrm{n}+1} \mathrm{CH}\left(\mathrm{CH}_{2}\right)_{2} \mathrm{COOH}$
FIG. 4. The Structures of Fatty Acids Present in the Sebum of Goats.

total fatty acids, were identified as oddcarbon-numbered anteiso-acids and evencarbon-numbered iso-acids by the comparison of gas chromatograms of their methyl esters with those of standard acids purified from lanolin. Moreover, none of the free acids obtained from the female extracts had such an intense odor as those from mature male goats. As methyl hexadecenoate and methyl octadecenoate overlapped on methyl esters of 14methylpentadecanoic acid and 16-methylheptadecanoic acid on gas chromatograms, re- spectively, the percentages of both unsaturated esters were calculated after hydrogenation of the same sample in the presence of platinum dioxide as a catalyst.

The series of the above methyl-branched fatty acids were also observed in the sebum of juveniles of both sexes. There was little sexual difference in the acidic constituents, and the normal fatty acids were the major ones in the total fatty acids. The chemical structures of the aforementioned fatty acids are illustrated in Fig. 4.

From these results, it is obvious that 4-ethyl fatty acids are the male-specific substances and that secretion of these acids is controlled by the hypothalamus-gonadal systems. ${ }^{1)}$ On occasion, small amounts of ethyl-branched fatty acids were detected on the female's body, just as is seen in the middle graph of Fig. 3 (young adult). These fatty acids were assumed to have come to her body surface from the mature males in her neighborhood, since the female reared in an isolated paddock did not have any ethyl analogs.

There are big differences in constituents of the sebum between sexes or ages rather than between breeds. It is interesting that, in both sexes, juveniles secrete the lipids mainly consisting of normal fatty acids, while branchedchain fatty acids become the major components in the acidic constituents of the sebum as the animals grow up. These changes suggest that the secretion is influenced by endocrine status; that is, the secretion of lipid from the sebaceous gland is considered to be controlled by sex hormones ${ }^{4)}$ and, in fact, this study revealed that the increase in the branchedchain fatty acids in the sebum was closely related to the sexual maturity in both males and females.

Various kinds of methyl-branched fatty acids were also recognized as minor components in the lipids of milk $^{5}$ and adipose tissue $^{6)}$ of ruminants and they are thought to have come from rumen microbial lipids. The origins of the branched fatty acids we found in the sebum may be different from those in body and milk lipids, and L-valine and L-isoleucine 
are considered to be indirect precursors of even-carbon-numbered iso-acids and oddcarbon-numbered anteiso-acids as disciussed for the lipids of lambs. ${ }^{7)}$

In preliminary studies, we have obtained results suggesting that 4-ethyl fatty acids specific to the mature male are not only a releaser pheromone but also a primer pheromone. The details will be published later.

Acknowledgment. This work was supported by a Grant-in-Aid for the Scientific Research from the Ministry of Education, Science and Culture of Japan.

\section{REFERENCES}

1) H. Sasada, T. Sugiyama, K. Yamashita and J. Masaki, Jpn J. Zootech. Sci., 54, 401 (1983), and references cited therein.

2) T. Sugiyama, H. Sasada, J. Masaki and K. Yamashita, Agric. Biol. Chem., 45, 2655 (1981).

3) A. W. Witkam, J. Am. Chem. Soc., 67, 447 (1945).

4) D. M. Stoddart, "Mammalian Odours and Pheromones," Arnold, London, 1976.

5) A. M. Massart-Leen, H. DePooter, M. Decloedt and N. Schamp, Lipids, 16, 286 (1981).

6) W. R. H. Duncan and G. A. Garton, Br. J. Nutr., 40, 29 (1978).

7) D. T. Downing, J. Lipid Res., 5, 210 (1964) 